
\title{
Original
article \\ Sexual relationships, risk behaviour, and condom use in the spread of sexually transmitted infections to heterosexual men
}

\author{
B A Evans, R A Bond, K D MacRae
}

Objective: To examine the effect of patient defined non-regular sexual relationships and other risk behaviours on the incidence of sexually transmitted infections in heterosexual men and the role of condom use in the prevention of their spread.

Design: A prospective cross sectional study of sexual behaviour reported by a standardised self administered questionnaire in new patients who presented for screening and diagnosis.

Setting: A genitourinary medicine clinic in west London.

Subjects: 957 consecutive newly attending heterosexual men who completed a sexual behaviour questionnaire in 1993/94.

Main outcome measures: Variables relating to sociodemographic status, sexual behaviour, condom use, sexually transmitted infections and testing for HIV infection, stratified by the reporting of non-regular partners.

Results: We found that the $65 \%$ of men who reported non-regular sexual partners were more likely to be white collar class $(\mathrm{d}=7 \cdot 5 \%, 95 \% \mathrm{CI}=1 \cdot 3,13 \cdot 7)$ and to have had sexual intercourse with non-United Kingdom born women ( $d=7 \cdot 8 \%, 95 \% \mathrm{CI}=3 \cdot 5,12 \cdot 2)$. They also reported coitarche before 16 years of age $(\mathrm{d}=13 \cdot 4 \%, 95 \% \mathrm{CI}=8 \cdot 0,18 \cdot 8)$ and many more sexual partners both in the last year $(\mathrm{d}=13 \cdot 1 \%, 95 \% \mathrm{CI}=10 \cdot 2,16 \cdot 0)$ and in their lifetime $(\mathrm{d}$ $=27 \cdot 9 \%, 95 \% \mathrm{CI}=21 \cdot 6,34 \cdot 2)$. They were significantly more likely to practise anal intercourse $(\mathrm{d}=8 \cdot 7 \%, 95 \% \mathrm{CI}=3 \cdot 3,14 \cdot 1)$, to smoke $(\mathrm{d}=16 \cdot 3 \%, 95 \% \mathrm{CI}=9 \cdot 8,22 \cdot 6)$, to drink alcohol $(\mathrm{d}=4 \cdot 9 \%, 95 \% \mathrm{CI}=1 \cdot 2,8 \cdot 6)$, and to have chlamydial infection $(\mathrm{d}=5 \cdot 7 \%, 95 \% \mathrm{CI}=$ $2 \cdot 2,9 \cdot 2$ ), of which $30 \%$ was subclinical. Increasing condom use with regular partners correlated with decreasing incidence of urethral infection (gonorrhoeal and/or chlamydial infection) $(p<0.03)$ and candidal balanitis $(p<0.03)$ and a greater likelihood of no infection being detected $(p=0.0002)$. Use of condoms with non-regular partners was much more frequent than with regular partners $(d=21 \cdot 4 \%, 95 \% \mathrm{CI}=16 \cdot 7,26 \cdot 1)$. However, we found evidence of oral transmission of urethral gonorrhoea and chlamydial infection among men who reported always using condoms. HIV infection was found in only two men $(0 \cdot 2 \%)$, both of whom reported intercourse with non-United Kingdom born women.

Conclusions: Heterosexual men who reported non-regular sexual relationships compensated for their increased risk lifestyle by using condoms more frequently and showed only an increased incidence of chlamydial infection. More consistent condom use with regular partners was significantly associated with the absence of sexually transmitted infection. These findings suggest that transmission between regular partners has been underestimated.

(Genitourin Med 1997;73:368-372)

Keywords: sexual behaviour; risk behaviour; condoms

\author{
Department of \\ Genitourinary \\ Medicine, Charing \\ Cross Hospital, \\ Fulham Palace Road, \\ London W6 8RF \\ B A Evans \\ Charing Cross and \\ Westminster Medical \\ School, Charing Cross \\ Hospital, London \\ W6 8RF \\ W6 8RF \\ K D MacRae \\ Correspondence to: \\ Dr B A Evans. \\ Accepted for publication \\ 20 May 1997
}

\section{Introduction}

In a previous paper on women presenting at a genitourinary medicine clinic in west London, ${ }^{1}$ we reported that regular, rather than non-regular, heterosexual partnerships played the major role in transmission of bacterial sexually transmitted infections and that non-regular partners appeared to present much less risk. Three explanations were proposed for these findings. These were that women are much more frequently exposed to genital infection through their regular partners, regular relationships change with time and, perhaps most important of all, only $20 \%$ of women never used condoms with non-regular partners.

In this paper we examine the same variables in relation to men who were newly attending the same genitourinary medicine clinic between 1 and 2 years later.

\section{Methods}

This study is based on a standardised self administered questionnaire given to all newly attending male patients. The first part concerned consent for HIV testing and risks of infection and the second part was devoted to sexual orientation and heterosexual behaviour. Variables of sexual behaviour sought were age at first intercourse, numbers of female partners in the past year and in total, the practices of oral and anal intercourse, condom use with regular and (if applicable) non-regular partners, cigarette smoking, and alcohol consumption. Patients were asked to complete a separate question on condom use with nonregular partners only if they had had nonregular partners. Those who did not complete this question were classified as having no nonregular partners. Sociodemographic data were 
Table 1 Non-regular sexual partners, sociodemographic characteristics, and sexual behaviour in heterosexual men

\begin{tabular}{|c|c|c|c|}
\hline Variables & $\begin{array}{l}\text { Non-regular } \\
\text { partners } \\
(n=623)(\%)\end{array}$ & $\begin{array}{l}\text { No non-regular } \\
\text { partners } \\
(n=334)(\%)\end{array}$ & $\begin{array}{l}\text { Difference (\%) } \\
(95 \% \text { CI) }\end{array}$ \\
\hline \multicolumn{4}{|l|}{ Age (years): } \\
\hline $13-19$ & $23(3 \cdot 7)$ & $11(3 \cdot 3)$ & $0 \cdot 4(-2 \cdot 0,2 \cdot 8)$ \\
\hline $20-24$ & $150(24 \cdot 1)$ & $71(21 \cdot 3)$ & $2 \cdot 8(-2 \cdot 7,8 \cdot 3)$ \\
\hline $25-29$ & $191(30 \cdot 7)$ & $99(29 \cdot 6)$ & $1 \cdot 1(-5 \cdot 1,7 \cdot 1)$ \\
\hline $30+$ & $259(41 \cdot 6)$ & $153(45 \cdot 8)$ & $-4 \cdot 2(-10 \cdot 8,2 \cdot 4)$ \\
\hline \multicolumn{4}{|c|}{$(n=612)$} \\
\hline white & $482(78 \cdot 8)$ & $266(80 \cdot 4)$ & $-1 \cdot 6(-7 \cdot 0,3 \cdot 8)$ \\
\hline black & $106(17 \cdot 3)$ & $50(15 \cdot 1)$ & $2 \cdot 2(-2 \cdot 7,7 \cdot 1)$ \\
\hline Asian & $14(2 \cdot 3)$ & $11(3 \cdot 3)$ & $-1 \cdot 0(-3 \cdot 3,1 \cdot 2)$ \\
\hline \multicolumn{4}{|l|}{ Marital status: } \\
\hline Single & $496(80 \cdot 4)$ & $244(74 \cdot 2)$ & $6 \cdot 2(0 \cdot 6,11 \cdot 9)$ \\
\hline Married & $69(11 \cdot 2)$ & $59(17 \cdot 9)$ & $-6.7(-11.6,-1.9)^{\star}$ \\
\hline Separated/divorced & $51(8 \cdot 3)$ & $23(7 \cdot 0)$ & $1 \cdot 3(-2 \cdot 2,4 \cdot 8)$ \\
\hline \multicolumn{4}{|l|}{ Socioeconomic class: } \\
\hline Professional & $122(21 \cdot 4)$ & $98(31 \cdot 6)$ & $-10 \cdot 2(-16 \cdot 3,-4 \cdot 0)^{\star \star}$ \\
\hline White collar & $184(32 \cdot 3)$ & $77(24 \cdot 8)$ & $7 \cdot 5(1 \cdot 3,13 \cdot 7)$ \\
\hline Blue collar & $102(17 \cdot 9)$ & $52(16 \cdot 8)$ & $1 \cdot 1(-4 \cdot 1,6 \cdot 4)$ \\
\hline Unemployed & $86(15 \cdot 1)$ & $38(12 \cdot 3)$ & $2 \cdot 8(-1 \cdot 8,7 \cdot 6)$ \\
\hline Student & $68(12 \cdot 0)$ & $41(13 \cdot 2)$ & $-1 \cdot 2(-5 \cdot 7,3 \cdot 3)$ \\
\hline \multicolumn{4}{|l|}{ Smoking: } \\
\hline nil & $313(50 \cdot 2)$ & $222(66 \cdot 5)$ & $-16 \cdot 3(-22 \cdot 6,-9 \cdot 8)^{\star \star \star}$ \\
\hline 1-10/day & $159(25 \cdot 5)$ & $61(18 \cdot 2)$ & $7 \cdot 2(1 \cdot 9,12 \cdot 6)$ \\
\hline$>10 /$ day & $151(24 \cdot 2)$ & $51(15 \cdot 3)$ & $8 \cdot 9(3 \cdot 9,14 \cdot 1)^{\star}$ \\
\hline \multicolumn{4}{|l|}{ Alcohol: } \\
\hline nil & $33(5 \cdot 3)$ & $34(10 \cdot 2)$ & $-4.9(-8.6,-1.2)^{\star}$ \\
\hline weekly or less & $278(44 \cdot 6)$ & $169(50 \cdot 6)$ & $-6 \cdot 0(-12 \cdot 6,0 \cdot 7)$ \\
\hline more than weekly & $312(50 \cdot 1)$ & $131(39 \cdot 2)$ & $10 \cdot 9(4 \cdot 3,17 \cdot 4)^{\star}$ \\
\hline \multicolumn{4}{|l|}{ Coitarche (years): } \\
\hline $6-15$ & $190(30 \cdot 5)$ & $57(17 \cdot 1)$ & $13 \cdot 4(8 \cdot 0,18 \cdot 8)^{\star \star \star}$ \\
\hline 16 & $103(16 \cdot 5)$ & $35(10 \cdot 5)$ & $6 \cdot 0(1 \cdot 7,10 \cdot 4)$ \\
\hline 17 & $115(18 \cdot 5)$ & $67(20 \cdot 1)$ & $-1.6(-6.9,3.7)$ \\
\hline 18 & $90(14 \cdot 4)$ & $64(19 \cdot 2)$ & $-4 \cdot 8(-9 \cdot 8,0 \cdot 3)$ \\
\hline $19-30$ & $125(20 \cdot 1)$ & $111(33 \cdot 2)$ & $-13 \cdot 1(-19 \cdot 1,-7 \cdot 2)$ \\
\hline \multicolumn{4}{|l|}{ Partners in past year: } \\
\hline $0-1$ & $147(23 \cdot 6)$ & $243(72 \cdot 8)$ & $-49 \cdot 2(-55 \cdot 0,-43 \cdot 3)$ \\
\hline 2 & $\begin{array}{l}186(29.9) \\
203(32.6)\end{array}$ & $\begin{array}{l}62(18 \cdot 6) \\
26(7 \cdot 8)\end{array}$ & $\begin{array}{l}11 \cdot 3(5 \cdot 8,16 \cdot 8) \\
24 \cdot 8(20 \cdot 1,29 \cdot 5)\end{array}$ \\
\hline $3-5$ & $\begin{array}{r}203(32 \cdot 6) \\
87(14 \cdot 0)\end{array}$ & $\begin{array}{r}26(7 \cdot 8) \\
3(0 \cdot 9)\end{array}$ & $24 \cdot 8(20 \cdot 1,29 \cdot 5)$ \\
\hline$\stackrel{>5}{\text { Partners in lifetime. }}$ & $87(14 \cdot 0)$ & $3(0.9)$ & $13 \cdot 1(10 \cdot 2,16 \cdot 0)^{\star \star \star}$ \\
\hline \multicolumn{4}{|l|}{ Partners in lifetime: } \\
\hline $\begin{array}{l}1 \\
2-5\end{array}$ & $\begin{array}{c}3(0 \cdot 5) \\
110(17 \cdot 7)\end{array}$ & $135(40 \cdot 4)$ & $\begin{array}{r}-7 \cdot 0(-9 \cdot 9,-4 \cdot 1) \\
-22 \cdot 7(-28 \cdot 8,-16 \cdot 7)\end{array}$ \\
\hline $\begin{array}{l}2-5 \\
6-10\end{array}$ & $146(23 \cdot 4)$ & $72(21 \cdot 6)$ & $\begin{aligned}-22 \cdot 7 & (-28 \cdot 8,-16 \cdot 7) \\
1 \cdot 8 & (-3 \cdot 7,7 \cdot 4)\end{aligned}$ \\
\hline $\begin{array}{l}6-10 \\
>10\end{array}$ & $364(58 \cdot 4)$ & $102(30.5)$ & $\begin{array}{r}1 \cdot 8(-3 \cdot 7,7 \cdot 4) \\
27 \cdot 9(21 \cdot 6,34 \cdot 2)^{\star \star \star}\end{array}$ \\
\hline \multicolumn{4}{|l|}{ Anal intercourse: } \\
\hline penetration & $168(27 \cdot 0)$ & $61(18 \cdot 3)$ & $8 \cdot 7(3 \cdot 3,14 \cdot 1)^{\star}$ \\
\hline ejaculation & $90(14 \cdot 4)$ & $26(7 \cdot 8)$ & $6.6(2 \cdot 7,10 \cdot 6)^{\star}$ \\
\hline \multicolumn{4}{|l|}{ Oral intercourse: } \\
\hline fellatio & $561(90 \cdot 0)$ & $297(88.9)$ & $1 \cdot 1(-3 \cdot 0,5 \cdot 2)$ \\
\hline ejaculation & $389(62.4)$ & $183(54 \cdot 8)$ & $7 \cdot 6(1 \cdot 1,14 \cdot 2)$ \\
\hline cunnilingus & $512(82 \cdot 2)$ & $276(82 \cdot 6)$ & $-0 \cdot 4(-5 \cdot 5,4 \cdot 6)$ \\
\hline \multicolumn{4}{|l|}{ Vaginal intercourse: } \\
\hline$<1 /$ week & $179(28 \cdot 7)$ & $71(21 \cdot 3)$ & $7 \cdot 4(1 \cdot 8,13 \cdot 1)$ \\
\hline $1-3 /$ week & $245(39 \cdot 3)$ & $140(41.9)$ & $-2.6(-9 \cdot 1,4 \cdot 0)$ \\
\hline$>3 /$ week. & $199(31 \cdot 9)$ & $123(36 \cdot 8)$ & $-4 \cdot 9(-11 \cdot 2,1 \cdot 5)$ \\
\hline \multicolumn{4}{|l|}{ Condoms with } \\
\hline $\begin{array}{l}\text { regular partners: } \\
\text { never }\end{array}$ & $\begin{array}{l}(\mathrm{n}=613) \\
218(35 \cdot 6)\end{array}$ & $\begin{array}{l}(n=331) \\
159(48 \cdot 0)\end{array}$ & $-12.4(-19 \cdot 1,-5.9)^{\star}$ \\
\hline $\begin{array}{l}\text { never } \\
\text { occasionally/often }\end{array}$ & $299(48 \cdot 8)$ & $108(32 \cdot 6)$ & $16 \cdot 2(9 \cdot 7,22 \cdot 6)$ \\
\hline $\begin{array}{l}\text { olways } \\
\text { alwation }\end{array}$ & $96(15 \cdot 7)$ & $64(19 \cdot 3)$ & $-3.6(-8.8,1.5)$ \\
\hline \multicolumn{4}{|l|}{$\begin{array}{l}\text { Condoms with non- } \\
\text { regular partners: }\end{array}$} \\
\hline never & $87(14 \cdot 2)$ & - & $21 \cdot 4(16 \cdot 7,26 \cdot 1) \dagger$ \\
\hline occasionally/often & $293(47 \cdot 8)$ & - & $1 \cdot 0(-4 \cdot 6,6 \cdot 6) \dagger$ \\
\hline always & $233(38 \cdot 0)$ & - & $-22 \cdot 3(-27 \cdot 1,-17 \cdot 5) \dagger$ \\
\hline
\end{tabular}

extracted from the self completed registration document and clinical data were taken from the medical case notes. Socioeconomic class was defined by occupation and ethnicity by self classification into the 1991 United Kingdom census categories.

For admission to the study, patients were required to have fully completed the sexual behaviour questionnaire and to have undergone clinical examination and screening for sexually transmitted and other genital infections. The screening included urethral culture for Neisseria gonorrhoeae, EIA (Syva Diagnostics) for Chlamydia trachomatis with confirmation by immunofluorescence and serological tests for syphilis (STS). Non-gonococcal urethritis (NGU) was diagnosed microscopically by the examination of Gram stained urethral smears under oil immersion $(\times 100)$ objectives if more than five polymorphonu- clear leucocytes (PMN) were found in three successive fields. Diagnosis of candidal balanitis required confirmation by culture. Cultures for herpes simplex virus were undertaken if clinically indicated and testing for HIV infection was offered to all patients but carried out only with informed consent. Prepubertal and non-English speaking males were excluded from the study.

Statistical analysis employed sPSS- $\mathrm{X}$ software on a Sun 4/670 computer. Confidence intervals for differences between percentages were calculated, using the Confidence Interval Analysis programme published by the BMJ. Statistical significance was measured by the $\chi^{2}$ test for homogeneity with Yates's correction and the $\chi^{2}$ test for trend. This paper reports findings only in self defined heterosexual men.

\section{Results}

Between September 1993 and September 19941212 unselected consecutive newly attending male patients, who described themselves as heterosexual, were given a questionnaire on heterosexual and related behaviour. There were $126(10.4 \%)$ who did not complete the questionnaire in full, eight $(0.7 \%)$ who did not conform to the protocol for admission, and five $(0.4 \%)$ who refused. A total of $116(9.6 \%)$ had attended only for HIV testing and declined full clinical examination and screening for genital infection. Accordingly, $957(79 \cdot 8 \%)$ men were eligible for the study. These were subdivided into those who reported non-regular partners $(623,65 \%)$ and those who did not $(334,35 \%)$. The latter group therefore self assessed all their partners as regular.

SOCIODEMOGRAPHIC DATA

On bivariate analysis, non-regular partners were associated with white collar class $(\mathrm{d}=$ $7.5 \%, 95 \% \mathrm{CI}=1.3,13 \cdot 7)$, smoking ( $\mathrm{d}=$ $16.3 \%, 95 \%$ CI $=9 \cdot 8,26 \cdot 2$ ), and more frequent alcohol consumption ( $\mathrm{d}=10.9 \%, 95 \%$ $\mathrm{CI}=4 \cdot 3,17 \cdot 4)$. There was a negative association with marriage $(\mathrm{d}=-6 \cdot 7 \%, 95 \% \mathrm{CI}=$ $-11 \cdot 6,-1 \cdot 9)$ and with professional class $(\mathrm{d}=$ $-10 \cdot 2 \%, 95 \%$ CI $=-16 \cdot 3,-4 \cdot 0)$. These results are shown in table 1 , from where it can be seen that non-regular partners had no relation to age or ethnicity.

\section{SEXUAL BEHAVIOUR}

Table 1 also shows marked differences between the two groups with earlier coitarche $(\mathrm{d}=13.4 \%, 95 \% \mathrm{CI}=8.0,18.8)$ and many more sexual partners both in the last year $(\mathrm{d}=13.1 \%, 95 \% \mathrm{CI}=10.2,16.0)$ and in total $(d=27 \cdot 9 \%, \quad 95 \% \quad C I=21 \cdot 6,34 \cdot 2)$ among those who had non-regular partners. This group were more likely to have had anal intercourse $(\mathrm{d}=8 \cdot 7 \%, 95 \% \mathrm{CI}=3 \cdot 3,14 \cdot 1)$, and fellatio with ejaculation $(\mathrm{d}=7 \cdot 6 \%, 95 \%$ $\mathrm{CI}=1 \cdot 1,14 \cdot 2)$. They also used condoms more frequently with their regular partners (d $=12.4 \%, 95 \%$ CI $=5 \cdot 9,19 \cdot 1)$. Furthermore, only $14 \%$ never used condoms with their non-regular partners. Infrequent inter- 
Table 2 Non-regular sexual partners, HIV testing, and risk behaviour in heterosexual men

\begin{tabular}{lllr}
\hline & $\begin{array}{l}\text { Non-regular } \\
\text { partners } \\
(n=618)(\%)\end{array}$ & $\begin{array}{l}\text { No non-regular } \\
\text { partners } \\
(n=331)(\%)\end{array}$ & $\begin{array}{l}\text { Difference }(\%) \\
(95 \% \text { CI })\end{array}$ \\
\hline Variables & $312(50 \cdot 5)$ & $90(27 \cdot 2)$ & $23 \cdot 3(17 \cdot 1,29 \cdot 5)^{\star \star \star}$ \\
Consent to HIV test & $11 / 614(1 \cdot 8)$ & $2 / 328(0 \cdot 6)$ & $1 \cdot 2(-0 \cdot 2,2 \cdot 5)$ \\
Risks: & $22 / 608(3 \cdot 6)$ & $3 / 327(0 \cdot 9)$ & $2 \cdot 7(0 \cdot 9,4 \cdot 5)$ \\
$\quad$ Injecting drug use (IDU) & $6 / 613(1 \cdot 0)$ & $6 / 328(1 \cdot 8)$ & $-0 \cdot 8(-2 \cdot 5,0 \cdot 8)$ \\
Partner of IDU & $59 / 611(9 \cdot 7)$ & $14 / 327(4 \cdot 3)$ & $5 \cdot 4(2 \cdot 2,8 \cdot 6)^{\star}$ \\
Homosexual intercourse & $104 / 611(17 \cdot 0)$ & $30 / 327(9 \cdot 2)$ & $7 \cdot 8(3 \cdot 5,12 \cdot 2)^{\star}$ \\
Partner & $7 / 614(1 \cdot 1)$ & $1 / 328(0 \cdot 3)$ & $0 \cdot 8(-0 \cdot 2,1 \cdot 9)$ \\
$\quad$ African & $(n=609)$ & $263(81)$ & $-14 \cdot 1(-19)$ \\
$\quad$ Elsewhere abroad & $413(67 \cdot 8)$ & $8 \cdot 7,-8 \cdot 5)$ & $(-2 \cdot 0,2 \cdot 3)$ \\
Blood transfusion & $16(2 \cdot 6)$ & $50(15 \cdot 6)$ & $14 \cdot 0(8 \cdot 6,19 \cdot 4)^{\star \star \star}$ \\
Prediction of results: & $180(29 \cdot 6)$ & &
\end{tabular}

course was also more likely in this group ( $\mathrm{d}=$ $7 \cdot 4 \%, 95 \% \mathrm{CI}=1 \cdot 8,13 \cdot 1$ ).

\section{RISK BEHAVIOUR AND HIV TESTING}

Fifty per cent of men with non-regular partners consented to HIV testing compared with $27 \%$ of men with no non-regular partners (d $=23.3 \%, 95 \% \mathrm{CI}=17 \cdot 1,29 \cdot 5)$. Their significantly increased risks (table 2) consisted mainly of intercourse with African partners (d $=5 \cdot 4 \%, 95 \% \mathrm{CI}=2 \cdot 2,8 \cdot 6)$ or partners from elsewhere abroad ( $d=7 \cdot 8 \%, 95 \% \mathrm{CI}=3 \cdot 5$, $12 \cdot 2)$. Only two men out of 271 tested positive $(0.7 \%)$ and both had partners from abroad. Not all men who consented to testing were actually tested. Men who had no non-regular partners were more likely to feel sure that they were HIV negative before testing $(\mathrm{d}=14 \cdot 1 \%$, $95 \% \mathrm{CI}=8 \cdot 5,19 \cdot 7)$.

\section{SEXUALLY TRANSMITTED INFECTIONS}

Despite highly significant differences in sexual behaviour, the men who had non-regular partners were found to be at significantly increased risk only for chlamydial infection $(\mathrm{d}=5 \cdot 7 \%$, $95 \% \mathrm{CI}=2 \cdot 2,9 \cdot 2)$ and $\mathrm{NGU}(\mathrm{d}=6.5 \%$, $95 \% \mathrm{CI}=0.9,12 \cdot 2)$. Chlamydial infection without evidence of urethritis was found in 21 men $(3.4 \%)$ with non-regular partners, but in only three men $(0.9 \%)$ who had no non-regular

Table 3 Non-regular sexual partners and genital infections in heterosexual men

\begin{tabular}{lccc} 
Genital infections & $\begin{array}{l}\text { Non-regular } \\
\text { partners } \\
(n=623)(\%)\end{array}$ & $\begin{array}{l}\text { No non-regular } \\
\text { partners } \\
(n=334)(\%)\end{array}$ & $\begin{array}{l}\text { Difference (\%) } \\
(95 \% \text { CI) }\end{array}$ \\
\hline $\begin{array}{l}\text { Gonorrhoea } \\
\text { Chlamydial infection }\end{array}$ & $24(3 \cdot 9)$ & $9(2 \cdot 7)$ & $1 \cdot 2(-1 \cdot 1,3 \cdot 5)$ \\
Non-gonococcal & $71(11 \cdot 4)$ & $19(5 \cdot 7)$ & $5 \cdot 7(2 \cdot 2,9 \cdot 2)^{\star}$ \\
$\quad$ urethritis (NGU) & $177(28 \cdot 4)$ & $73(21 \cdot 9)$ & $6 \cdot 5(0 \cdot 9,12 \cdot 2)$ \\
Non-chlamydial NGU & $135(21 \cdot 7)$ & $58(17 \cdot 4)$ & $4 \cdot 3(-0 \cdot 9,9 \cdot 5)$ \\
Candidal balanitis & $30(4 \cdot 8)$ & $21(6 \cdot 3)$ & $-1 \cdot 5(-4 \cdot 6,1 \cdot 6)$ \\
Genital herpes & $25(4 \cdot 0)$ & $19(5 \cdot 7)$ & $-1 \cdot 7(-4 \cdot 6,1 \cdot 3)$ \\
Genital warts & $65(10 \cdot 4)$ & $53(15 \cdot 9)$ & $-5 \cdot 4(-10 \cdot 0,-0 \cdot 8)$ \\
No abnormality detected & $197(31 \cdot 6)$ & $109(32 \cdot 6)$ & $-1 \cdot 0(-7 \cdot 2,5 \cdot 2)$ \\
\hline
\end{tabular}

${ }^{\star} \mathrm{p}<0.01$.

Table 4 Non-regular sexual partners and past history of genital infections

\begin{tabular}{lccc}
\hline Genital infections & $\begin{array}{l}\text { Non-regular } \\
\text { partners } \\
(n=623)(\%)\end{array}$ & $\begin{array}{l}\text { No non-regular } \\
\text { partners } \\
(n=334)(\%)\end{array}$ & $\begin{array}{l}\text { Difference }(\%) \\
(95 \% C I)\end{array}$ \\
\hline Gonorrhoea & $32(5 \cdot 1)$ & $14(4 \cdot 2)$ & $0 \cdot 9(-1 \cdot 8,3 \cdot 7)$ \\
Chlamydial infection & $19(3 \cdot 0)$ & $9(2 \cdot 7)$ & $0 \cdot 3(-1 \cdot 8,2 \cdot 6)$ \\
NGU & $99(15 \cdot 9)$ & $48(14 \cdot 4)$ & $1 \cdot 5(-3 \cdot 2,6 \cdot 3)$ \\
Candidal balanitis & $16(2 \cdot 5)$ & $10(3 \cdot 0)$ & $-0 \cdot 5(-2 \cdot 6,1 \cdot 8)$ \\
Genital herpes & $19(3 \cdot 0)$ & $15(4 \cdot 5)$ & $-1 \cdot 5(-4 \cdot 0,1 \cdot 2)$ \\
Genital warts & $27(4 \cdot 3)$ & $21(6 \cdot 3)$ & $-2 \cdot 0(-5 \cdot 0,1 \cdot 1)$ \\
No past history & $355(57 \cdot 0)$ & $197(59 \cdot 0)$ & $-2 \cdot 0(-8 \cdot 6,4 \cdot 6)$ \\
\hline
\end{tabular}

partners. The corresponding figures for dual infection with gonorrhoea were eight men and one man respectively. Therefore, chlamydial infection was subclinical in $21(33 \%)$ of 63 men who had reported non-regular partners and in three $(16.7 \%)$ of 18 men who had not. The proportions with non-chlamydial NGU did not differ significantly between the two groups $(\mathrm{d}=4.3 \%, 95 \% \mathrm{CI}=-0.9,9.5)$. Paradoxically, genital warts were significantly more frequent in men who reported no nonregular partners $(\mathrm{d}=-5.4 \%, 95 \% \mathrm{CI}=$ $-10.0,-0.8)$. More than one diagnosis was made in 116 men $(18.6 \%)$ in the non-regular partner group and in 48 men $(14.4 \%)$ in the rest (table 3).

The effects of non-regular partners in terms of past history of genital infection are shown in table 4. We found no significant differences between the two groups of patients, therefore, in any previous genital infections related to sexual intercourse.

\section{CONDOM USE}

The four point self assessment scale for condom use ("never", "occasionally", “often", "always") was analysed for specific infections by the $\chi^{2}$ test for trend, both for use with regular and non-regular partners (Table 5).

The reporting of increasing condom use with regular partners was associated with significant downtrends in the incidence of gonorrhoea and/or chlamydial infection $(\mathrm{p}<0.03)$ and in the incidence of candidal balanitis $(p<0.03)$ and a highly significant uptrend in Oo the proportion of men who had no genital $N$ infection $(p=0.0002)$. Increasing condom $\mathrm{c}$ use with non-regular partners showed no significant differences in the proportion of men with any of the common genital infections or $\mathbb{D}$ with no genital infection. However, condom use with non-regular partners was much more frequent with only $14 \%$ never using condoms and $38 \%$ always using condoms ( $p<0.00001)$.

Reported condom use with all sexual partners failed to prevent three cases of urethral gonorrhoea $(9 \cdot 1 \%)$ and five cases of chlamydial infection $(5 \cdot 6 \%)$. The clinical records of these patients were re-examined: of the three patients with gonorrhoea, one gave a consistent clinical history of never using condoms, one had had only fellatio (4 days previously) in the past month and in the third condoms were ineffective for reasons which were not 
Table 5 Condom use with regular and non-regular partners and genital infections in heterosexual men (1993/94)

\begin{tabular}{|c|c|c|c|c|c|c|c|}
\hline & \multicolumn{4}{|l|}{ Condom use } & \multicolumn{3}{|c|}{$\chi^{2}$ for trend } \\
\hline & $\begin{array}{l}\text { Never } \\
(\%)\end{array}$ & $\begin{array}{l}\text { Occasionally } \\
(\%)\end{array}$ & $\begin{array}{l}\text { Often } \\
(\%)\end{array}$ & $\begin{array}{l}\text { Always } \\
(\%)\end{array}$ & $\chi^{2} T$ & $p$ Value & $\begin{array}{l}\text { Never versus always } \\
\text { Diff \% }(95 \% \text { CI) }\end{array}$ \\
\hline $\begin{array}{l}(\mathrm{n}=249) \\
\text { Non-chlamydial NGU }(\mathrm{n}=192) \\
\text { Candidal balanitis }(\mathrm{n}=51) \\
\text { Genital herpes }(\mathrm{n}=44) \\
\text { Genital warts }(\mathrm{n}=118) \\
\text { NAD }(\mathrm{n}=299)\end{array}$ & $\begin{array}{l}102(27 \cdot 1) \\
76(20 \cdot 2) \\
27(7 \cdot 2) \\
20(5 \cdot 3) \\
50(13 \cdot 3) \\
92(24 \cdot 4)\end{array}$ & $\begin{array}{l}81(29 \cdot 2) \\
65(23 \cdot 5) \\
14(5 \cdot 1) \\
16(5 \cdot 8) \\
33(11 \cdot 9) \\
92(33 \cdot 2)\end{array}$ & $\begin{aligned} & 33(25 \cdot 4) \\
& 23(17 \cdot 7) \\
& 6(4 \cdot 6) \\
& 2(1 \cdot 5) \\
& 11(8 \cdot 5) \\
& 53(40 \cdot 8)\end{aligned}$ & $\begin{array}{l}33(20 \cdot 6) \\
28(17 \cdot 5) \\
4(2 \cdot 5) \\
6(3 \cdot 8) \\
24(15 \cdot 0) \\
62(38 \cdot 8)\end{array}$ & $\begin{array}{l}2 \cdot 25 \\
0 \cdot 76 \\
4 \cdot 93 \\
1 \cdot 74 \\
0 \cdot 0001 \\
15 \cdot 76\end{array}$ & $\begin{array}{l}0.13 \\
0.39 \\
<0.03 \\
0.18 \\
0.99 \\
0.0002\end{array}$ & $\begin{array}{c}6 \cdot 5(-1 \cdot 3,14 \cdot 1) \\
2 \cdot 7(-4 \cdot 5,9 \cdot 8) \\
4 \cdot 7(1 \cdot 1,8 \cdot 2) \\
1 \cdot 5(-2 \cdot 2,5 \cdot 3) \\
-1 \cdot 7(-8 \cdot 2,4 \cdot 8) \\
-14 \cdot 4(-23 \cdot 1,-5 \cdot 6)\end{array}$ \\
\hline $\begin{array}{l}\text { Non-regular partners: } \\
\text { Gonorrhoea }(\mathrm{n}=24) \\
\text { Chlamydial infection }(\mathrm{n}=71) \\
\text { Either/both }(\mathrm{n}=87) \\
\text { Non-gonococcal urethritis (NGU) }\end{array}$ & $\begin{array}{l}n=87 \\
3(3 \cdot 4) \\
10(11 \cdot 5) \\
12(13 \cdot 8)\end{array}$ & $\begin{array}{l}n=143 \\
9(6 \cdot 3) \\
20(14 \cdot 0) \\
25(17 \cdot 5)\end{array}$ & $\begin{array}{l}n=157 \\
3(1 \cdot 9) \\
21(13 \cdot 4) \\
23(14 \cdot 6)\end{array}$ & $\begin{array}{l}n=236 \\
9(3 \cdot 8) \\
20(8 \cdot 5) \\
27(11 \cdot 4)\end{array}$ & $\begin{array}{l}0 \cdot 31 \\
1 \cdot 61 \\
1 \cdot 32\end{array}$ & $\begin{array}{l}0 \cdot 58 \\
0 \cdot 20 \\
0 \cdot 25\end{array}$ & $\begin{array}{c}-0 \cdot 4(-4 \cdot 9,4 \cdot 2) \\
3 \cdot 0(-4 \cdot 6,10 \cdot 6) \\
2 \cdot 4(-6 \cdot 0,10 \cdot 7)\end{array}$ \\
\hline $\begin{array}{l}(\mathrm{n}=177) \\
\text { Non-chlamydial NGU }(\mathrm{n}=135) \\
\text { Candidal balanitis }(\mathrm{n}=30) \\
\text { Genital herpes }(\mathrm{n}=25) \\
\text { Genital warts }(\mathrm{n}=65) \\
\text { NAD }(\mathrm{n}=197)\end{array}$ & $\begin{array}{l}25(28 \cdot 7) \\
20(23 \cdot 0) \\
4(4 \cdot 6) \\
7(8 \cdot 0) \\
6(6 \cdot 9) \\
24(27 \cdot 6)\end{array}$ & $\begin{array}{l}38(26 \cdot 6) \\
30(21 \cdot 0) \\
4(2 \cdot 8) \\
5(3 \cdot 5) \\
15(10 \cdot 5) \\
43(30 \cdot 1)\end{array}$ & $\begin{array}{l}48(30 \cdot 6) \\
31(19 \cdot 7) \\
7(4 \cdot 5) \\
7(4 \cdot 5) \\
18(11 \cdot 5) \\
54(34 \cdot 4)\end{array}$ & $\begin{array}{l}66(28 \cdot 0) \\
54(22 \cdot 9) \\
15(6 \cdot 4) \\
6(2 \cdot 5) \\
26(11 \cdot 0) \\
76(32 \cdot 2)\end{array}$ & $\begin{array}{l}0 \cdot 006 \\
0 \cdot 02 \\
1 \cdot 44 \\
3 \cdot 46 \\
0 \cdot 83 \\
0 \cdot 72\end{array}$ & $\begin{array}{l}0.94 \\
0.89 \\
0 \cdot 23 \\
0.06 \\
0.36 \\
0 \cdot 40\end{array}$ & $\begin{array}{l}0.9(-10 \cdot 3,11 \cdot 9) \\
0.1(-10 \cdot 2,10.4) \\
-1.8(-7 \cdot 2,3 \cdot 6) \\
5 \cdot 5(-0.6,11 \cdot 6) \\
-4 \cdot 1(-10 \cdot 8,2 \cdot 5) \\
-4.6(-15 \cdot 7,6.5)\end{array}$ \\
\hline
\end{tabular}

recorded. This also applied to four patients with chlamydial infection, but the fifth gave a history of fellatio only with a prostitute 3 weeks previously.

\section{Discussion}

A positive response to condom use with nonregular partners proved to be a powerful discriminator of many aspects of heterosexual behaviour in heterosexual men, very similar to our findings in heterosexual women. ${ }^{1}$ Both men and women who had non-regular partners reported earlier coitarche, many more partners and were more likely to practise both oral and anal intercourse. They also used condoms more frequently with their regular partners and were more likely to be smokers. However, non-regular partners were reported by $65 \%$ of men compared with $44 \%$ of women. Paradoxically, this apparently increased risk behaviour correlated only with a greater likelihood of chlamydial infection, a condition frequently asymptomatic in both sexes. Excluding coexisting urethral gonorrhoea, $24(29.6 \%)$ of 81 men with chlamydial infection had no evidence of urethritis on urethral smear.

The effect of increasing condom use also correlated with a declining incidence of bacterial infections in both sexes. Urethral infection with either chlamydia or gonorrhoea or both decreased with increasing condom use. In heterosexual men, this also applied to candidal balanitis and the absence of any genital infection $(p=0.0002)$. In women, we found that it applied to trichomoniasis. ${ }^{1}$ No significant trends were observed for condom use with non-regular partners apart from an increase in the incidence of genital warts among women, which could have been a consequence of their antecedent presence.

Condom benefit against gonorrhoea and NGU has recently been reported by Cates and Holmes $^{2}$ who re-examined data collected in $1975 .^{3}$ Male volunteers were interviewed on return to ship after shore leave about their sexual behaviour and use of condoms. Fourteen per cent of non-users subsequently developed gonorrhoea or NGU but no men who reported condom use $(p=0.02)$. In our study, both these infections were acquired by a small percentage of men despite reportedly consistent condom use for vaginal intercourse. No explanation for this could be elicited on retrospective scrutiny in five out of the eight case histories. One patient ticked "always" in the questionnaire, but consistently indicated at interview that he did not use condoms. Two patients, one with gonorrhoea and one with chlamydial infection, had practised only oropenile intercourse within the relevant incubation periods, thus suggesting a small but definite risk of transmission from pharynx to urethra. Nevertheless, condom use had failed to prevent transmission of infection by vaginal intercourse in $3 \%$ of men with gonorrhoea and $4.4 \%$ of men with chlamydial infection, assuming the information given was correct.

We found a large discrepancy between the number of men who consented to HIV testing (402) and the number actually tested $(271-67 \%)$. Of those tested, only two $(0.7 \%)$ were positive, but both had high risk histories. However, no less than $73 \%$ of the 930 responders felt sure they were negative in the first place, so that given the labour intensive nature of HIV testing, it comes as no surprise that this was not undertaken in one third of those who had previously consented. Perhaps the time has come to include HIV testing in routine screening for STI in heterosexual men who have no high risk behaviour.

A report based on data from the National Study on Sexual Attitudes and Lifestyles (NSSAL) ${ }^{4}$ found that men and women at notionally increased risk of STI were more likely to have attended a GUM clinic. For example, $28 \%$ of their population sample of men who had had both more than five female sexual partners and paid for sex had attended a GUM clinic in the past 5 years compared with $3.4 \%$ overall. However, no information was available on the presence or absence of an STI. The study therefore showed that people who believed themselves to have practised risk 
behaviour attended GUM clinics. This may only serve to perpetuate misconceptions about what constitutes risk-for example, how great a risk is payment for sex?

Our studies provide evidence that men and women at potential risk of acquiring STI adopt behaviour to minimise the risk. Our data point to chlamydial infection, perhaps the least symptomatic bacterial STI, as the greatest risk and confirm the protective effect of condom use. This awareness leading to appropriately adaptive behaviour seems the most likely explanation for the apparent paradox of our findings. STI are transmitted in ignorance rather than knowingly between regular partners with whom there is most frequent contact and least apparent risk.

1 Evans BA, Kell PD, Bond RA, MacRae KD. Heterosexual relationships and condom-use in the spread of sexually transmitted diseases to women. Genitourin Med 1995; 71:291-4

2 Cates W, Holmes KK. Re: condom efficacy against gonorrhoea and nongonococcal urethritis. Am $\mathcal{f}$ Epidemiol 1996;143:843-4.

3 Hooper RR, Reynolds GH, Jones OG, Zaidi A, Wiesner PJ, Latimer KP, et al. Cohort study of venereal disease. The risk of gonorrhoea transmission from infected women to men. Am F Epidemiol 1978;108:136-44.

4 Johnson AM, Wadsworth J, Wellings $K$, Field J. Who goes to STD clinics? Results from a national population survey. Genitourin Med 1996;72:197-202. 